\title{
BORIS VIAN, UN PRÉCURSEUR DE L'OULIPO"
}

\author{
Beatriz Vaz Leão**
}

\begin{abstract}
Résumé: Ce travail a pour but de définir ce que l'on appelle OULIPO et, par la suite, de montrer pourquoi Boris Vian peut être considéré comme un précurseur de ce groupe littéraire. Pour ce faire, nous prendrons comme exemple son oeuvre L'Arrache-coeur où nous chercherons à montrer le travail qu'il fait du point de vue du langage.
\end{abstract}

L'OULIPO, OUvroir de LIttérature POtentielle, n'est pas, comme l'a dit son fondateur Raymond Queneau, un mouvement littéraire. Il s'agit d'un groupe d'écrivains qui se sont réunis pour la première fois en 1960 , et qui continuent de se réunir, dans le but d'explorer méthodiquement et systématiquement les potentialités de la littérature ou, plus généralement, de la langue. Mais cette exploitation des potentialités de la littérature ne se fait pas n'importe comment. Les écrivains, pour mener à bien cette tâche, s'assignent certaines contraintes auxquelles ils tiennent beaucoup. Parmi ces contraintes, qui ne sont pas suivies par tous les écrivains oulipiens de manière ortodoxe, on peut citer comme exemple

\footnotetext{
- Recebido para publicação em novembro 2004.

* Professora da Faculdade de Letras/UFMG
} 
celles qui portent sur la création de structures, sur la création de mots nouveaux, sur l'élimination d'une voyelle, susceptibles toutes de permettre la production d'oeuvres originales.

Il faut dire que, dans cette recherche, certains concepts mathématiques, tel l'utilisation de ressources de la combinatoire, jouent un rôle important. D'ailleurs, le co-fondateur de ce groupe, François Le Lionais, est un mathématicien qui s'intéresse beaucoup à la littérature. Ce que le groupe avait l'intention d'accentuer, c'est le lien qui existe entre la littérature et les mathématiques. Au cœur de la démarche oulipienne trône donc une contrainte qui ne s'assimile pas aux règles de grammaire.

Les écrivains oulipiens, en fait, ne font que jouier avec la langue, mais ce jeu n'a rien à avoir avec ce que faisaient les écrivains surréalistes. Au contraire, il s'agit là d'une remise en question, en littérature, du travail surréaliste, puisque les contraintes sont choisies d'avance, de manière consciente. Les écrivains oulipiens se distinguent aussi des écrivains existentialistes, car ils ne prêchent pas d'engagement de tỵpe sartrien.

Parmi les contraintes créées par les écrivains oulipiens, on peut citer l'anagramme, le lipogramme, le chronogramme, etc. Ces contraintes, apparemment paradoxales, ne bloquent pas l'imagination; au contraire, elles l'éveillent et la stimulent. La pratique oulipienne est donc une pratique consciente, assez souvent collective, et toutes les règles auxquelles les écrivains oulipiens se soumettent sont remplies de sens.

Boris Vian est né en 1917 et il est mort très jeune, à l'âge de 39 ans. Il a exercé plusieurs métiers. Ingénieur des Arts et Manufactures, passionné des mathématiques, il a été aussi chanteur, comédien, chansonnier, journaliste, traducteur, mécanicien, menuisier, peintre et écrivain.

Le goût du ludique, qu'il a manifesté d'une manière générale dans son œuvre, pourrait être un des principes moteurs 
de la création d'une littérature annonçant, pour ainsi dire, celle des écrivains oulipiens. Dans certaines de ses œuvres et, en particulier, dans L'Arrache-cœur, on peut remarquer des traits, des ébauches de contraintes, pourrait-on dire, bien proches de celles prêchées plus tard par les oulipiens. Néanmoins, différemment des œuvres des écrivains oulipiens, les nouveautés créées par Boris Vian ne sont pas systématiques.

Dans L'Arrache-coeur , l'histoire est construite autour de deux destins parallèles: celui de Clémentine et celui de Jacquemort.

L'histoire se passe dans un village normand et, en particulier; dans une maison où vivent Clémentine et son mari Angel. Clémentine vient d'accoucher de trois enfants, les "trumeaux", à qui elle donne les noms de Noël, Joël et Citroën. Révoltée contre les souffrances de la grossesse et de l'accouchement, elle chasse son mari de la maison et s'occupe de ses enfants de manière cruelle et même monstrueuse. Obsédée par l'amour maternel, Clémentine voit les enfants comme des objets qui lui appartiennent. Elle s'imagine toute sorte de danger auquel les enfants peuvent être confrontés et finit par les enfermer dans des cages dorées afin de les protéger et de les avoir toujours auprès d'elle.

Jacquemort, un psychiatre aux caractéristiques bizarres, arrive au village le jour même de l'accouchement et il vient aider Clémentine en sage-femme. Invité à s'installer dans la maison, il essaie d'analyser le comportement des gens. Il s'agit d'un personnage vide, sans existence intérieure, qui s'enrichit, qui se nourrit des rêves, des pensées, des craintes de la vie des autres. Il va souvent au village et il devient transparent quand il cesse de désirer les envies des autres, c'est-à-dire, d'absorber les pensées et les gestes des villageois, et même celle d'un chat.

Au fil de ses promenades, Jacquemort découvre que les villageois ont des mœurs assez singulières; leur morale est bien différente de celle que nous connaissons et que nous pratiquons 
d'habitude car elle est fondée sur l'absence de toute honte. On voit donc, comme une chose très naturelle, des villageois qui maltraitent les enfants, les vieillards et les animaux. Un habitant nommé la Gloïre, qui passe ses journées dans une barque, flottant dans un ruisseau du village, est payé en or pour garder toute la honte de la communauté et il passe son temps à pêcher toutes les ordures que les habitants du village jettent dans la rivière. Jacquemort, après avoir essayé de psychanalyser plusieurs villageois, finit par accepter de prendre la place de la Gloïre.

On peut trouver aussi dans le roman des personnages assez pittoresques tels qu'un maréchal-ferrant qui, tout en étant amoureux de Clémentine, fait pourtant l'amour avec un automate dans son lieu de travail; Clémentine, elle, ressent à ce même instant, la jouissance de cet acte d'amour vécu par le maréchal, bien qu'elle soit seule à la maison; une couturière aux yeux peints et qui cout sans rien voir, un curé qui menace les villageois et qui prêche une religion fondée sur l'argent, l'or et le luxe; des paysans et des artisans mal nourris, exploités et qui sont maltraités comme des bêtes.

On perçoit, dans L'Arrache-coeur, une critique sousjacente aux idées des surréalistes qui accordent une grande importance aux apports de la psychanalyse. Le personnage principal, comme nous l'avons dit, est un psychanaliste, né adulte, portant une notice: "Psychiatre. Vide. A remplir." Depuis son arrivée dans le village, ce qu'il cherche c'est des gens à psychanalyser. En bavardant avec Angel et en lui posant des questions sur la possibilité de trouver des gens à psychanalyser, celui-ci lui dit:

"- Il y en a plein, dit Angel. Vous aurez la nurse quand vous voudrez. Et les gens du village ne refuseront pas. 
Ce sont des gens un peu grossiers, mais intéressants et riches." (VIAN, 1962:24)

A ces mots, Jacquemort ajoute:

"Je suis vide. Je n'ai que gestes, réflexes, habitudes. Je veux me remplir. C'est pourquoi je psychanalyse les gens. Mais mon tonneau est un tonneau des Danaïdes. Je n'assimile pas. Je leur prends leurs pensées, leurs complexes, leurs hésitations, et rien ne m'en reste. Je n'asssimile pas." (VIAN, 1962: 24)

Boris Vian se moque également de l'existentialisme sartrien quand le personnage Jacquemort affirme qu'il n'est pas né libre, ce qui suppose qu'il a déjà un destin à accomplir:

“(...) Je suis né l'année dernière, tel que vous me voyez devant vous. Regardez ma carte d'identité. (....) 'Psychiatre. Vide. A remplir.' Une notice! C'est indiscutable. (...) Alors, vous voyez bien que ça ne vient pas de moi, ce désir de me remplir, dit Jacquemort. Que c'était joué d'avance. Que je n'étais pas libre." (VIAN, 1962:26)

Et en fait, à la fin du roman il sera condamné à remplacer La Glö̈re dans le ruisseau du village. On y perçoit donc un certain refus du compromis, de l'engagement, de la responsabilité. En effet, le supposé engagement de Jacquemort ne serait simplement que l'acceptation du destin qui lui avait été conféré.

Peu à peu, on commence à voir les liens existants entre les deux personnages, La Gloïre et Jacquemort. Tout comme le Christ, ils sont chargés de sauver le monde. La Gloïre se nourrit d'or et porte la honte des villageois, tandis que Jacquemort se livre à psychanalyser les gens pour en absorber leurs pensées, 
leurs fautes, leurs rêves. Il est donc prêt à prendre la honte de toute la communauté sur ses épaules.

Un autre trait commun aux écrivains oulipiens qui apparait assez souvent dans L'Arrache-coeur, bien que ce ne soit pas de manière systématique et méthodique, c'est l'utilisation de divers jeux de langage. Dans le roman, on constate la présence de néologismes, des confessions nettes et claires sur le travail conscient de l'écriture. Nos remarques se porteront, ainsi, sur l'orthographe, le lexique, la syntaxe et la composition.

Le moyen de déformation graphique le plus fréquent dans le roman est le tréma, utilisé sur des noms propres et des noms communs, parfois de manière différente de là norme orthographique, dans le but de déformer le monde de façon humoristique. Nous savons que le tréma s'emploie en français sur les voyelles e, i, u, pour indiquer que la voyelle précédente doit être prononcée séparément. Dans L'Arracbe-coeur, le personnage La Glö̈re - une parodie du Christ traversant la mer sans se mouiller - traverse un ruisseau rouge, dans une barque, sans se mouiller également.

"Le ruisseau l'intriguait. D'abord, il n'y avait rien eu, puis, tout à coup, il coulait large, plein jusqu'au bord, comme sous une membrane tendue. De la couleur de la bave du crache-sang, rouge claire et opaque (...)." (VIAN, 1962: 31)

“(...) il vit l'homme s'accrocher au bord et s'efforcer d y remonter. L'eau du ruisseau rouge passait sur ses vêtements, en perles vives, sans le mouiller(...). C'était un homme âgé. Il avait un visage creusé, les yeux bleus lointains." (VIAN, $1962: 47)$

D'autres exemples où l'utilisation du tréma est un écart par rapport à la règle se trouvent dans les noms de certains 
habitants du village - Chärles, Lalouët, Chrëtien, Nüfaire - et dans certains noms communs comme par exemple progrës, hömme de terre. Les noms de personnes nous font penser à des déformations conscientes:

Chärles $\rightarrow$ allusion à des rois français

Chrëtien $\rightarrow$ allusion à la religion

Nüfaire $\rightarrow$ allusion à celui qui ne fait rien

Lalouët $\rightarrow$ qui rapelle un sobriquet faisant allusion à un oiseau: l'alouette

Il en est de même pour le nom commun "progrës"' où la notion de progrès est mise en question.

En ce qui concerne le lexique, ce sont les néologismes de L'Arrache-coeur qui nous frappent le plus. Le narrateur-personnage les emploie, par exemple, pour désigner des espèces végétales qu'il retrouve dans le jardin, autour de la maison: Cormarin rappelle romarin, sirte rappelle myrthe, garillia semble un mélange de garrique et dahlia, senciaire fait penser à un mélange de sensitive et de cinéraire. Ce qui est intéressant de remarquer, c'est que dans le monde humain, les villageois présentent des comportaments inhumains, tandis que la nature est humanisée.

«Jacquemort avançait sans se presser et regardait les calamines dont le coeur rouge sombre battait au soleil. A chaque pulsation, un nuage de pollen s'élevait, puis retombait sur les feuilles agitées d'un lent tremblement.» (VIAN, 1962: 9)

Sur la route se trouvent également des espèces insolites telles les brouillouses, les arioles ou encore, les mailorties, celles-ci décrites comme étant des plantes sauvages, vertes, urticantes et filassouses. 
Une innovation très intéressante se trouve aussi dans les deux propositions synonymes créées par Boris Vian où le nom "maréchal-ferrant" est décomposé, à tour de rôle, chacun des éléments nominaux devenant un verbe dont l'autre est le sujet: marechala le ferrant et ferranta le maréchal. L'effet des sens produit est celui de rudesse, ce qui caractérise le personnage du roman.

Quant à la syntaxe, ce qui attire l'attention du lecteur chez Boris Vian, c'est la conscience qu'il a des jeux de langage dont il se sert pour montrer un monde dérisoire.

L'Arrache-coeur est constitué de chapitres datés chacun à la manière d'un journal et les 14 premiers chapitres ont des dates similaires à celles d'un calendrier normal. Mais à partir du $15 \mathrm{e}$ chapitre, on remarque un changement dans les dates et dans le numérotage des chapitres qui ne sont plus en ordre. Le temps semble s'étirer et se contracter différemment, les jours s'accumulent, laissant chez le lecteur l'impression d'un grand trouble mental. Les dates telles 27 juinet, 39 juinoût, 55 janvril, 73 févrin, 67 novrier, 79 décars, etc témoignent une destruction et une reconstruction de la langue, volontaires, créant des néologismes pour suggérer ce que ce monde avait d'étrange. Au tout début du chapitre 15, Boris Vian explique son travail avec le langage, en donnant la clé de ses procédés de création:

"Tant avait des fois Jacquemort pris le chemin du village qu'il lui était devenu aussi plat qu'un couloir d'asile et aussi nu qu'un barbu rasé. Un simple chemin, une voie comme une ligne est une ligne, sans épaisseur n'existe pas. Et raccourci se trouvait ce chemin. Pieds connus, pas déjà faits (pas de marche et non de négation). Embrouiller, devait-il, inverser, mais c'est insuffisant, les mêler, mieux, de parasites lettriques et logiques pour la parcourir, la route, sans ennui, ses pensées simples. Et tout de même 
il arrivait au bout chaque fois. Il chantait aussi:

Le chant du canon,

Le chant du départ,

Le chandelle nazalle,

Le chancre moule,

Le chacre mouille,

La chambre mousse." (VIAN,1962:112-113)

Remarquons que les verbes embrouiller, inverser, $\underline{\text { mêler, }}$ indiquent les procédés utilisés par Boris Vian et, d'autre part, annoncent, presque d'une manière prophétique, ceux qui seraient utilisés plus tard par les écrivains oulipiens. Boris Vian embrouille la phrase et la pensée, inverse des constituants. Au lieu de dire Jacquemort avait pris le chemin tant de fois, il dit «tant avait de fois Jacquemort pris le chemin...». Puis, il mêle les composants de mots divers, leur ajoute «des parasites lettriques et logiques», ce qui annonce aussi un des procédés chers aux écrivains oulipiens.
«L'aubépine, c'est une fleur
La graisse, c'est du gras
La m..., c'est du bonheur
Jésus, c'est mieux que tout ça.
La viande, c'est pour papa
Les cheveux, c'est pour la tête
Jésus, c'est du rabiuxe
Jésus, c'est un pluxe
Jésus, c'est un luxe... " (VIAN, $1962: 85)$

Après des vers naïfs, soit en ce qui concerne la métrique, la rime et les strophes, tous formés de mots très simples, il introduit le mot Jésus, qui, dans les deux avant derniers vers, sera défini par des néologismes contenant le mot «luxe». On y voit l'intention de l'auteur de montrer les attitudes de certains gens d'église, ce qui se confirme dans une phrase prononcée, 
à un moment donné, par le curé : «ma religion restera un luxe.»

Comme j'espère avoir montré, le travail de l'écriture est une des constantes dans le roman L'Arrache-coeur de Boris Vian. Par des procédés tels que la greffe de plusieurs mots, l'inversion de structure, la création de néologismes, Boris Vian peut être considéré un précurseur de l'Oulipo.

Selon FOURNEL (1981:284), «l'objetif des ateliers de l'Oulipo n'est pas de 'fabriquer' des écrivains. Mais plutôt de faire que, grâce aux bienfaits de la contrainte, on puisse écrire en jouant.» L'écriture devient un jeu. Bien que Boris Vian ne l'ait pas fait de manière systématique, on remarque chez lui la présence de contraintes oulipiennes auxquelles il tient, pour produire, de manière consciente, une littérature ludique. En prêchant la déconstruction du monde, à savoir, le déconstruction du langage, Boris Vian, dans le roman L'Arrache-coeur, finit par construire un poème en prose, pour chanter cette liberté de l'écriture, une écriture faite de jeux sans revendications, et aussi d'invention sans illogisme.

Resumo: Este trabalho tem por objetivo definir em que consiste o OULIPO e, em seguida, mostrar por qual razão podemos considerar Boris Vian como um precursor desse grupo literário. Para isto, partimos da obra L'Arrache-coeur onde procuramos mostrar o trabalbo que Boris Vian faz com a linguagem.

\section{Références bibliographiques}

BENS, Jacques. Boris Vian. Paris: Bordas, 1976.

FOURNEL, Paul. In : Clés pour la litterature potentielle. Paris: Nadaud, 1972. 
LAPPRAND, Marc. Poétique de lOulipo. Paris: Rodopi, 1998.

OULIPO, Atlas de littérature pontentielle. Paris: Gallimard, 1981.

VIAN, Boris. L'Arracbe-coeur. Paris: Christian Bourgois editeur, 1962. 